\title{
ESTUDO DA CIRCULAC̣̃̃O HEPATOMESENTÉRICA PELA ANGIOGRAFIA POR RESSONÂNCIA MAGNÉTICA COM GADOLÍNIO: COMPARAÇÃO ENTRE DOSES SIMPLES E DUPLA NO ESTUDO DE PACIENTES ESOUISTOSSOMÓTICOS*
}

\author{
Rogério Pedreschi Caldana ${ }^{1}$, Alexandre Sérgio de Araújo Bezerra ${ }^{2}$, Giuseppe D'Ippolito ${ }^{3}$, Jacob Szejnfeld ${ }^{4}$
}

Resumo OBJETIVO: Determinar a freqüência de visualização dos segmentos da circulação hepatomesentérica pela angiografia por ressonância magnética (angio-RM) com contraste e comparar o valor do método, utilizandose duas diferentes dosagens de gadolínio (doses simples e dupla). MATERIAIS E MÉTODOS: Estudo prospectivo de 36 pacientes esquistossomóticos submetidos a angio-RM. Os exames foram realizados em equipamento de RM de 1,5 T, usando-se bobina de corpo e bomba injetora para a administração endovenosa do contraste. Foram utilizadas, de maneira randomizada, dose dupla do contraste paramagnético $(0,2 \mathrm{mmol} / \mathrm{kg}$ de Gd-DTPA) em 21 pacientes e dose simples $(0,1 \mathrm{mmol} / \mathrm{kg})$ em outros 15 pacientes. Os exames foram interpretados por dois observadores em consenso, que classificaram o grau de visualização de 25 segmentos vasculares estabelecidos para análise, sem conhecimento da dose de gadolínio utilizada. RESULTADOS: Os segmentos vasculares proximais e de maior calibre foram as estruturas com melhor grau de visualização na maioria da amostra em estudo. 0 tronco celíaco, a artéria hepática comum, a artéria esplênica, a croça e terço médio da artéria mesentérica superior, a veia porta, a veia esplênica e a veia mesentérica superior apresentaram grau 2 de visualização em mais de $70 \%$ da amostra. Quanto à comparação das diferentes dosagens, não houve diferença significante $(p<0,05)$ no grau de visualização das diversas estruturas analisadas entre os grupos dose simples e dose dupla, com uma exceção isolada: na avaliação da artéria hepática direita $(p=0,008)$, o grupo dose simples apresentou maior freqüência de visualização grau 2 , com valor significante. CONCLUSÃO: 0 grau de visualização dos segmentos vasculares hepatomesentéricos pela angio-RM com contraste é elevado, sendo maior nos segmentos proximais e de maior calibre. A comparação entre os grupos que utilizaram dose simples e dupla de contraste demonstrou resultados semelhantes.

Unitermos: Angiografia por ressonância magnética; Esquistossomose; Gadolínio.

Abstract Gadolinium-enhanced magnetic resonance angiography for hepatomesenteric vascular evaluation: single and double doses comparison in schistosomiasis patients.

OBJECTIVE: To evaluate the visibility of hepatomesenteric vascular segments by 3D gadolinium-enhanced magnetic resonance (MR) angiography and to compare the method effectiveness between two different gadolinium doses (single and double doses). MATERIALS AND METHODS: A prospective study was performed with 36 schistosomiasis patients who were submitted to 3D contrast-enhanced MR angiography. Scans were performed in a high-field equipment $(1.5 \mathrm{~T})$, with body coil and power injector for intravenous contrast administration. Contrast double doses (Gd-DTPA $0.2 \mathrm{mmol} / \mathrm{kg}$ ) and single doses $(0.1 \mathrm{mmol} / \mathrm{kg}$ ) were randomly used respectively in 21 and 15 patients. Studies were interpreted by consensus between two observers who have rated the visualization degree of 25 proximal vascular segments without knowing the dose used. RESULTS: Proximal and calibrous vascular segments have presented higher visualization degree in the greatest part of the sample studied. The celiac trunk, common hepatic artery, splenic artery, proximal and medium third of superior mesenteric artery, portal vein, splenic vein and superior mesenteric vein have presented grade 2 visualization in more than $70 \%$ of the sample studied. As regards comparison between different doses, there was no significant difference $(p<0.05)$ in the visualization degree of several structures evaluated, between double dose and single dose groups, except for an isolate case of evaluation of right hepatic artery $(p=0.008)$ in which the single dose group has presented a higher frequency of grade 2 visualization with statistical significance. CONCLUSION: The visualization degree of hepatomesenteric vascular segments by 3D gadolinium-enhanced MR angiography is high, especially in the proximal and calibrous segments. The comparison between groups using single and double contrast doses has demonstrated similar results. Keywords: Magnetic resonance angiography; Schistosomiasis; Gadolinium.

\footnotetext{
* Trabalho realizado no Departamento de Diagnóstico por Imagem da Universidade Federal de São Paulo-Escola Paulista de Medicina, São Paulo, SP.

1. Doutorado pelo Departamento de Diagnóstico por Imagem da Universidade Federal de São Paulo-Escola Paulista de Medicina.

2. Pós-graduando do Departamento de Diagnóstico por Imagem da Universidade Federal de São Paulo-Escola Paulista de Medicina.

3. Professor Adjunto do Departamento de Diagnóstico por Imagem da Universidade Federal de São Paulo-Escola Paulista de Medicina.

4. Professor Livre-Docente do Departamento de Diagnóstico por Imagem da Universidade Federal de São Paulo-Escola Paulista de Medicina.

Endereço para correspondência: Dr. Rogério Pedreschi Caldana. Rua Joaquim Távora, 1299, ap. 82, Vila Mariana. São Paulo, SP, 04038-002. E-mail: rogercal@uol.com.br

Recebido para publicação em 1/8/2005. Aceito, após revisão, em 3/10/2005.
} 


\section{INTRODUÇÃO}

A partir de 1994, a introdução da angiografia por ressonância magnética (angioRM) com gadolínio endovenoso tornou disponível um novo e promissor método de estudo vascular ${ }^{(1,2)}$. Através de metodologia fundamentada em seqüências ultra-rápidas, associadas ao alto contraste fornecido pela primeira passagem vascular do gadolínio, encontrou-se um meio de estudar os vasos sanguíneos com qualidade comparável à imagem da angiografia convencional, com as vantagens da mínima invasividade e a não utilização do contraste iodado, potencialmente alérgeno e nefrotóxico. Por esses motivos, a angio-RM rapidamente ganhou espaço na literatura e na prática diagnóstica, tornando-se um dos principais objetos de estudo na área de RM na última década.

Inicialmente, inúmeros estudos procuraram validar este novo procedimento comparando-o com a arteriografia, considerada o método de referência para a avaliação vascular desde sua introdução no início da década de 1950. Nesse sentido, a angio-RM com gadolínio apresentou eficácia diagnóstica comparável à arteriografia quanto à identificação do grau e localização de estenoses nos territórios aortoilíaco ${ }^{(3-6)}$, renal $^{(7)}$ e ramos arteriais supra-aórticos ${ }^{(8,9)}$.

No território vascular mesentérico, a utilização da angio-RM com gadolínio foi demonstrada no ano de 2000 por Ernst et $a l .{ }^{(\mathbf{1 0})}$, em estudo comparativo entre a arteriografia e a angio-RM em 33 pacientes. A concordância obtida foi superior a $60 \%$ para a maioria dos segmentos vasculares, excetuando-se as artérias intra-hepáticas e ramificações da artéria mesentérica superior, que foram mais bem avaliadas pela arteriografia. Os resultados apresentados tiveram consistência pela natureza prospectiva e metodologia padronizada. No entanto, os autores utilizaram dose simples de contraste paramagnético em vez de dose dupla, fator que ainda não tem sustentação suficiente na literatura quanto aos resultados de visibilidade em comparação ao uso da dose dupla no território mesentérico.

De fato, a dose de gadolínio é uma das variáveis mais polêmicas em angio-RM e motivo de diversas pesquisas recentes. Com o intuito de maximizar o realce vas- cular, os primeiros trabalhos utilizaram dose dupla de contraste $(0,2 \mathrm{mmol} / \mathrm{kg})$, o que se tornou padrão no estudo dos diversos segmentos vasculares ${ }^{(\mathbf{1 , 2})}$. Também já foi demonstrado que o aumento da dose para o triplo do habitual $(0,3 \mathrm{mmol} / \mathrm{kg})$, apesar de seguro, não traz benefícios significativos para a qualidade da imagem em comparação à dose dupla ${ }^{(11)}$.

Em contrapartida, com o progressivo desenvolvimento de recursos técnicos e conseqüente ganho na resolução temporal e espacial das sequiências de exame, começou a ser investigada a eficácia da dose simples de contraste na angio-RM quando comparada à dose dupla, principalmente considerando-se a economia relacionada ao alto custo do contraste paramagnético ${ }^{(12)}$. Com esse intuito, foram realizados diversos estudos em alguns territórios arteriais, com bons resultados a favor da dose simples de gadolínio, como na avaliação da aorta abdominal $^{(12,13)}$, artérias renais ${ }^{(14-16)} \mathrm{e}$ artérias carótidas ${ }^{(17)}$.

O principal argumento a favor da dose dupla está relacionado à maior extensão do bolus de contraste, o que permitiria maior margem de erro no planejamento temporal do exame, podendo contribuir para a melhor qualidade da imagem em estudos prejudicados por outras variáveis. Além disso, o maior pico de realce vascular promovido pela dose dupla poderia eventualmente correlacionar-se à maior conspicuidade na avaliação de vasos de pequeno calibre, fator de relevância no território mesentérico. Entretanto, os ganhos tecnológicos recentes aumentaram a velocidade de aquisição dos dados ${ }^{(18,19)}$ e aprimoraram o contraste e o detalhamento da imagem. Isto reduziu o valor atribuído à dose dupla de contraste e tornou necessária sua reavaliação.

Com base nessas considerações e nos resultados publicados na literatura, questiona-se se o uso da dose dupla de gadolínio seria capaz de oferecer melhor visualização de segmentos da circulação hepática e mesentérica ou melhor reprodutibilidade do método, quando comparada à dose simples de contraste. Por outro lado, se os resultados entre as doses simples e dupla forem semelhantes, será possível reduzir a dose de contraste na angio-RM do território hepatomesentérico, diminuindo o custo do exame sem alteração da eficácia do método.
Com o intuito de avaliar a vascularização hepática e mesentérica através da angio-RM, procuramos um grupo homogêneo de pacientes, capaz de fornecer substrato consistente para tal pesquisa, e cujo próprio estudo traria também benefício diagnóstico para os indivíduos examinados. Por essas razões, optamos por estudar um grupo de pacientes esquistossomóticos.

A importância de se testar variáveis relacionadas à técnica de exame também neste grupo tem como proposta avançar mais uma etapa na consolidação da angioRM como método diagnóstico de grande refinamento técnico e aplicabilidade prática.

Diante do exposto, idealizamos este estudo com os objetivos de: a) determinar a freqüência de visualização dos segmentos da circulação hepatomesentérica através da angio-RM com contraste; b) comparar o valor do método entre a utilização de dose simples e dupla de contraste paramagnético.

\section{MATERIAIS E MÉTODOS}

Entre junho de 2000 e junho de 2002, realizamos trabalho prospectivo duplocego estudando a circulação hepatomesentérica de 36 pacientes esquistossomóticos de procedência ambulatorial, através da técnica de angio-RM tridimensional com gadolínio. Os critérios de inclusão no estudo foram: pacientes com idade superior a 18 anos, conscientes e colaborativos, com diagnóstico de esquistossomose mansônica por biópsia retal ou forte evidência clínicolaboratorial e epidemiológica (pacientes procedentes de área endêmica, com achados de imagem compatíveis com fibrose periportal e exame de fezes positivo). Estabelecemos os seguintes critérios de exclusão: contra-indicações ao exame de RM (marca-passo cardíaco, clipes de aneurisma cerebral, implantes cocleares, claustrofobia), antecedentes de etilismo (ingestão de etanol superior a $160 \mathrm{~g} / \mathrm{dia}$ ), sorologia positiva para vírus da hepatite $\mathrm{B}$ ou $\mathrm{C}$, antecedentes de doença auto-imune comprovada (pelo risco de hepatite auto-imune associada), e o uso conhecido de drogas hepatotóxicas.

O grupo estudado foi constituído por 19 homens e 17 mulheres, com idade entre 28 e 71 anos (mediana $=43$, média $=44,6$ ). 
Entre os 36 pacientes esquistossomóticos estudados, dez foram previamente submetidos a esplenectomia e os outros 26 pacientes apresentavam algum grau de esplenomegalia no momento do estudo (índice esplênico superior a 800), e não tinham antecedentes de cirurgia para derivação porto-sistêmica.

Os exames foram realizados em equipamento de RM modelo ACS/NT (Philips), operando em alto campo magnético $(1,5 \mathrm{~T})$ com gradiente de $15 \mathrm{mT} / \mathrm{m}$. Foi utilizada bobina de corpo para transmissão e recepção de sinais. Utilizamos bomba injetora modelo Spectris (Medrad) nos 14 primeiros casos da amostra e bomba modelo Injektron 82 MRT (Medtron) nos 22 casos restantes.

O exame abdominal foi realizado com as técnicas TSE e FFE, através de seqüências ponderadas em T1 em fase e oposição de fase, seqüências ponderadas em T2 com dois tempos de eco distintos (90 e $160 \mathrm{~ms}$ ) e seqüência axial ponderada em T1 após a injeção do contraste paramagnético. $\mathrm{O}$ estudo por angio-RM foi realizado com a sequiência FFE (eco gradiente), em três fases consecutivas obtidas com apnéia: sem contraste, arterial e venosa portal. O volume de aquisição tridimensional foi planejado no plano coronal, posicionado anteriormente à aorta, incluindo sua luz, e acompanhando a orientação de seu trajeto.

A injeção do contraste foi realizada através de bomba mecânica à velocidade constante de $3 \mathrm{ml} / \mathrm{s}$, seguida pela injeção de 15 a $20 \mathrm{ml}$ de solução fisiológica.

O tempo de intervalo para início da fase arterial da sequiência dinâmica foi calculado pelo método da dose teste, utilizandose $1 \mathrm{ml}$ de contraste seguido de $15 \mathrm{ml}$ de solução fisiológica. Este tempo variou, no grupo estudado, entre 9 e 21 segundos após o início da injeção. A fase venosa foi iniciada 60 segundos após o término da aquisição da fase arterial.

Utilizamos dose dupla do contraste paramagnético $(0,2 \mathrm{mmol} / \mathrm{kg}$, equivalente a $0,4 \mathrm{ml} / \mathrm{kg}$ de Gd-DTPA) em 21 pacientes, e dose simples $(0,1 \mathrm{mmol} / \mathrm{kg}$, equivalente a $0,2 \mathrm{ml} / \mathrm{kg}$ ) nos outros 15 pacientes. A decisão pela dose de contraste injetado foi feita por método de randomização, para

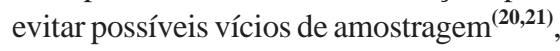
sendo estabelecida, por sorteio prévio, a seqüência de dosagens a ser utilizada pelos pacientes no estudo

Os exames foram reconstruídos pela técnica MIP, obtendo-se imagens tridimensionais em 16 projeções distintas. A análise dos exames foi baseada tanto nas imagens originais documentadas nas duas fases dinâmicas pós-subtração (arterial e portal) como nas imagens provenientes das reconstruções com técnica MIP (Figura 1).

A interpretação das imagens foi feita por dois examinadores em consenso, com experiência equivalente em RM e superior a quatro anos de trabalho na especialidade.

Foram estabelecidos 25 segmentos vasculares da circulação hepatomesentérica para análise do grau de visualização: tronco celíaco, artéria hepática comum, artéria hepática própria, artéria gástrica esquerda, artéria esplênica, artéria gastroduodenal, artéria gástrica direita, artéria hepática direita, artéria hepática esquerda, artéria hepática média, croça da artéria mesentérica superior, terço médio da artéria mesentérica superior, terço distal da artéria mesentérica superior, artérias jejunais e ileais, artéria cólica média, artéria cólica direita, artéria ileocólica, artéria pancreaticoduodenal inferior, artéria mesentérica inferior, veia porta, ramo portal direito, ramo portal esquerdo, veia esplênica, veia mesentérica superior e veia mesentérica inferior.

As estruturas identificadas foram graduadas de forma subjetiva com notas de 0 a 2, de acordo com seu grau de visualiza-

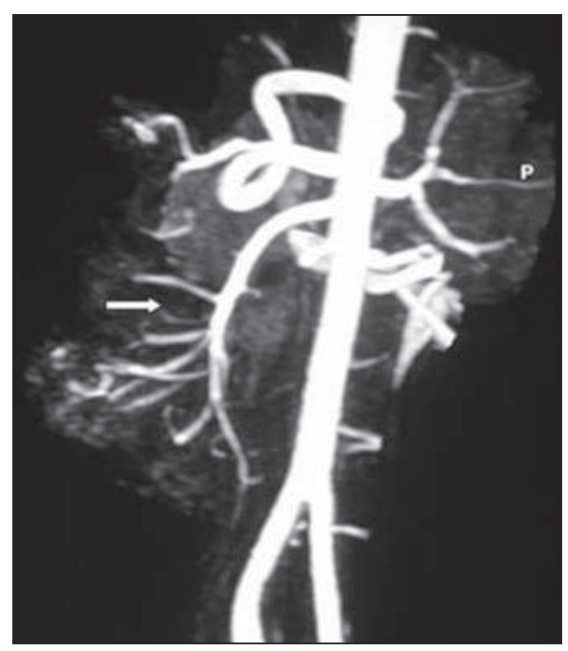

Figura 1. Exemplo de reconstrução da seqüência tridimensional pela técnica MIP na fase arterial. A artéria mesentérica superior e suas ramificações são identificadas pela seta. ção pela contrastação endovenosa: a nota 0 (zero) foi atribuída a estruturas não identificadas, a nota 1, para estruturas parcialmente visualizadas e nota 2 , para estruturas visualizadas com nitidez.

As Figuras 2 a 13 ilustram a visualização de alguns dos principais segmentos vasculares em estudo.

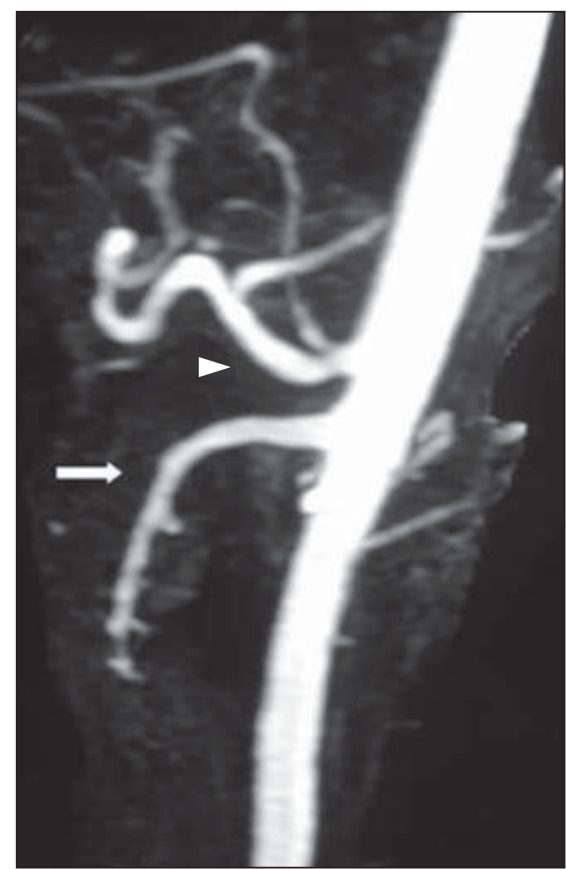

Figura 2. Reconstrução MIP demonstrando boa visualização do tronco celíaco (cabeça de seta) e artéria mesentérica superior nas regiões da croça e terço médio (seta).

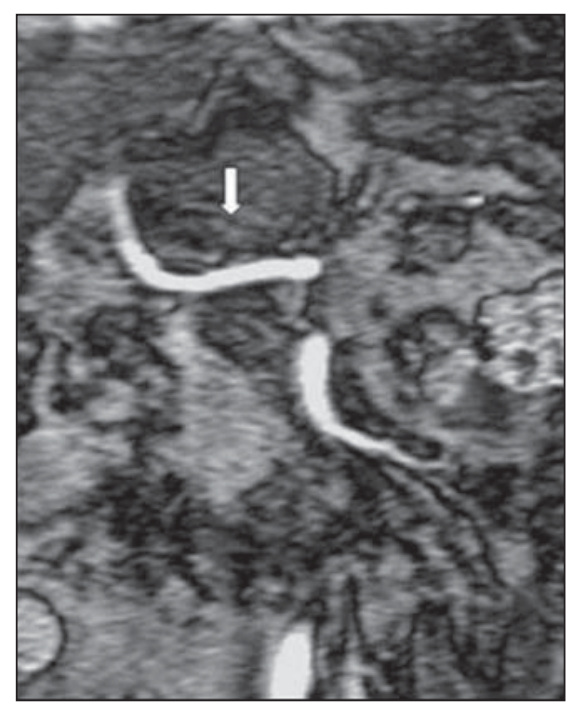

Figura 3. Visualização da artéria hepática comum (seta). 


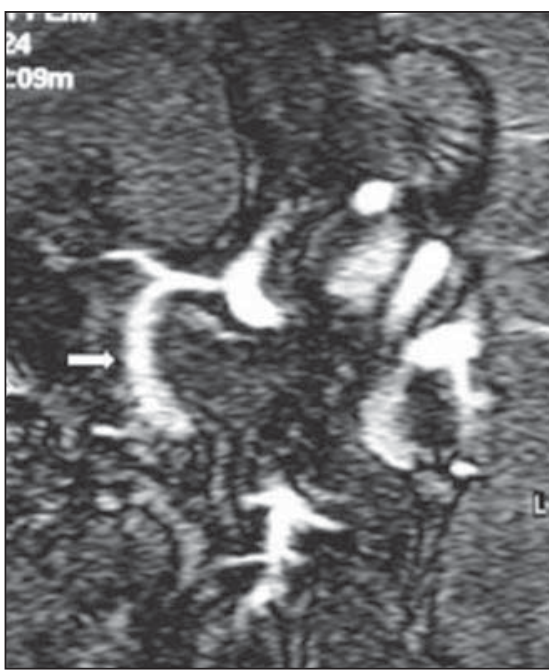

Figura 4. Visualização da artéria gastroduodenal (seta).

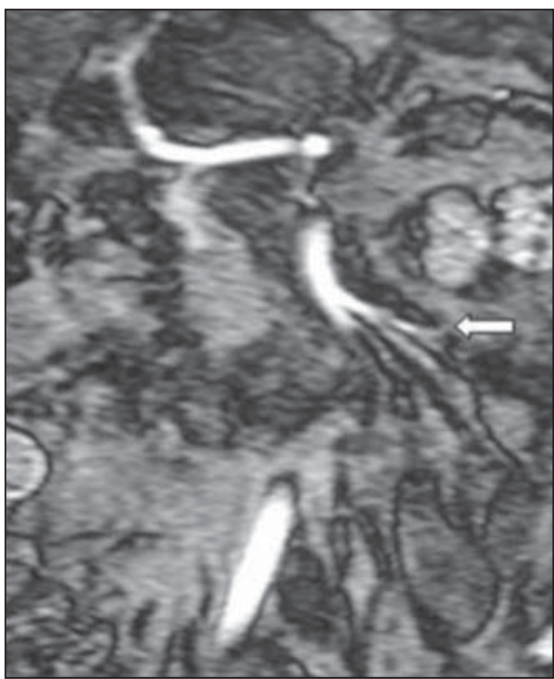

Figura 7. Exemplo da boa visualização (grau 2) de artérias jejunais (seta).

\section{Análise estatística}

A análise comparativa entre os grupos dose simples e dose dupla foi feita através da aplicação do teste de Fisher. O nível de significância foi estabelecido em 5\% ( $p<$ 0,05). Para permitir a aplicação do teste e simplificar a interpretação dos resultados, reduzimos as tabelas para apenas duas categorias de graus de visualização, agrupando os graus 0 e 1 em um único grupo. Este grupo reúne os casos cujo grau de visualização não tem qualidade suficiente para fins diagnósticos.

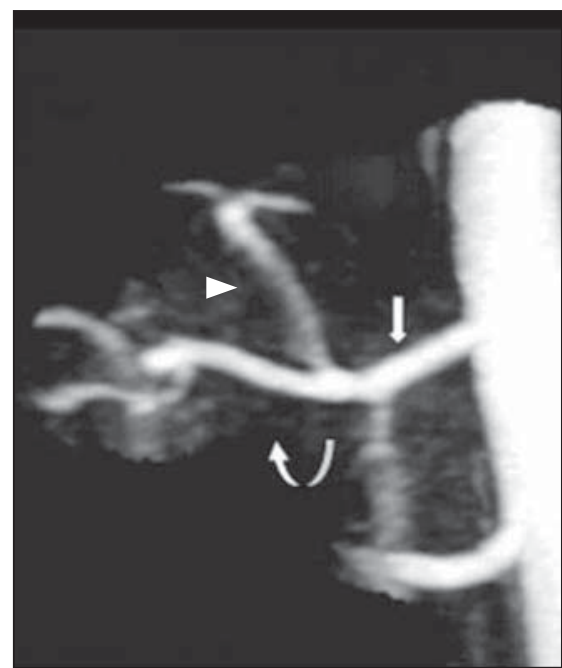

Figura 5. Imagem localizada de reconstrução MIP demonstrando as artérias hepáticas comum (seta), direita (seta curva) e esquerda (cabeça de seta).

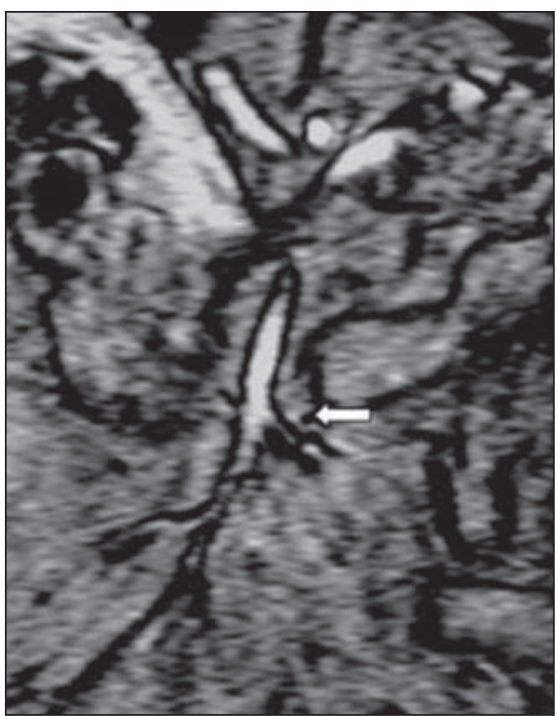

Figura 8. Exemplo de visualização com nitidez parcial (grau 1) de uma artéria jejunal/ileal (seta).

\section{RESULTADOS}

\section{Freqüência de visualização dos ramos vasculares}

Os segmentos vasculares proximais e de maior calibre foram as estruturas com melhor grau de visualização na maioria da amostra em estudo. O tronco celíaco, a artéria hepática comum, a artéria esplênica, a croça e terço médio da artéria mesentérica superior, a veia porta, a veia esplênica e a veia mesentérica superior apresentaram grau 2 de visualização em mais de $70 \%$ da

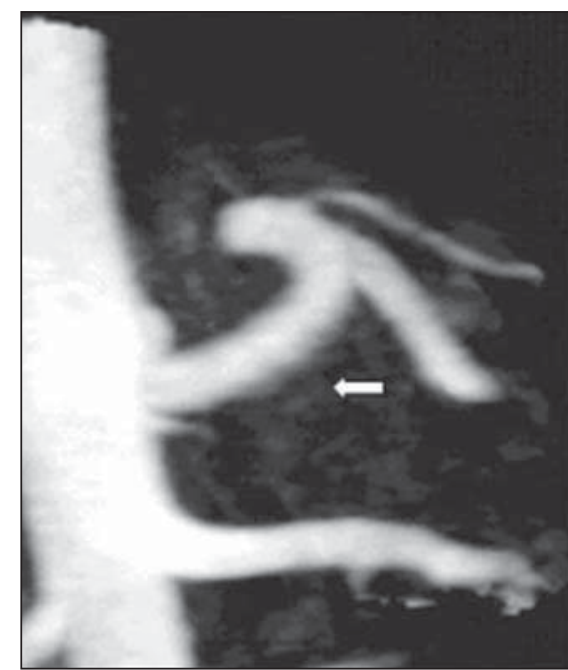

Figura 6. Visualização da artéria esplênica (seta) em imagem de reconstrução pela técnica MIP.

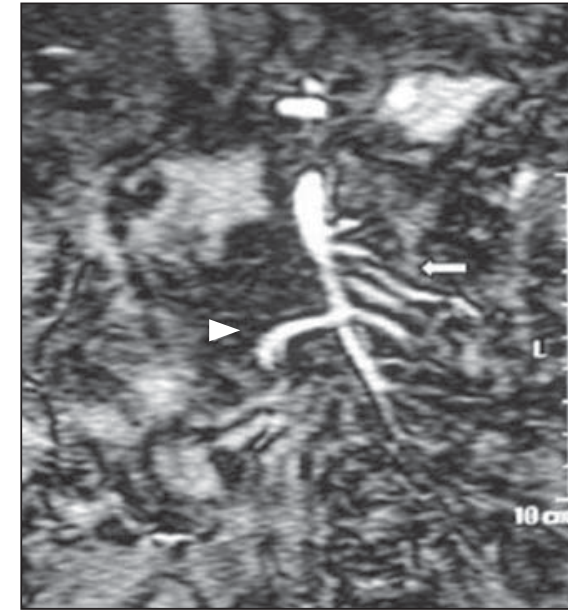

Figura 9. Visualização da artéria cólica direita (cabeça de seta) e artérias jejunais e ileais (seta).

amostra. Já a artéria gástrica direita, a artéria hepática média e a artéria pancreaticoduodenal inferior foram as estruturas com pior grau de visualização, não sendo identificadas em mais de $93 \%$ da amostra por ambos observadores. As Tabelas 1 e 2 apresentam a frequiência de visualização dos segmentos vasculares pelos dois observadores nos grupos dose simples e dose dupla de contraste.

$\mathrm{Na}$ amostra estudada, foram identificados sete casos de transformação cavernosa da veia porta, caracterizada por indefinição 


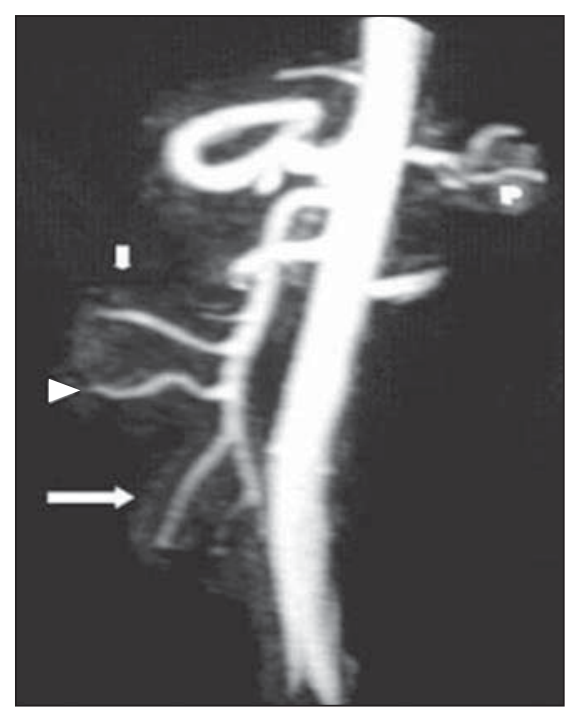

Figura 10. Visualização das artérias cólica direita (seta menor), cólica média (cabeça de seta) e ileocólica (seta maior) em imagem obtida por reconstrução MIP.

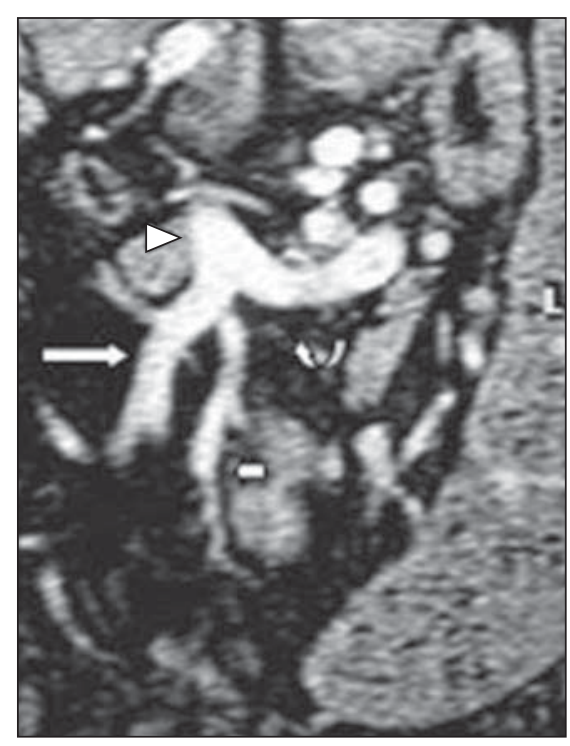

Figura 13. Visualização da veia mesentérica superior (seta maior), veia esplênica (seta curva), junção esplenomesentérica (cabeça de seta) e veia mesentérica inferior (seta menor), esta última tributária da veia mesentérica superior (variação anatômica).

dos contornos da veia porta associada à presença de estruturas venosas tortuosas ao longo do leito portal (Figuras 14 e 15).

Com exceção de um segmento vascular (artéria hepática direita), todos os demais segmentos estudados apresentaram grau de visualização semelhante entre os diferentes grupos de dosagem (diferença sem significância estatística). Isto pode ser observado nas Figuras 16 e 17. Apenas a artéria

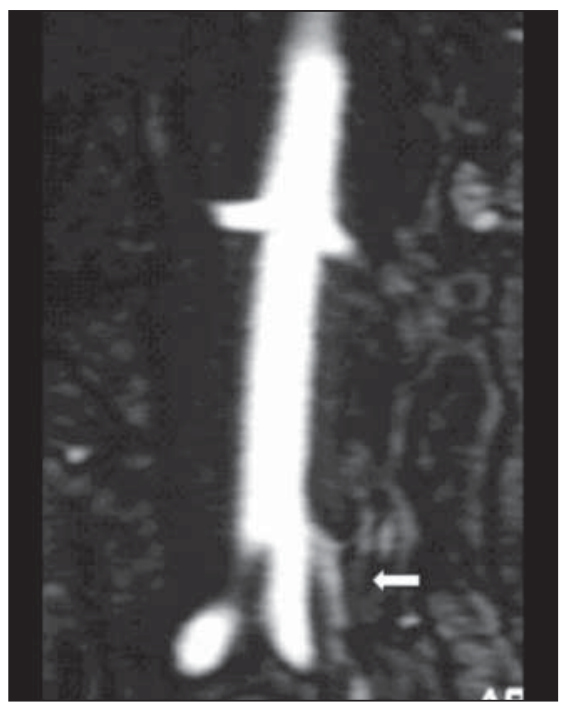

Figura 11. Visualização da artéria mesentérica inferior (seta).

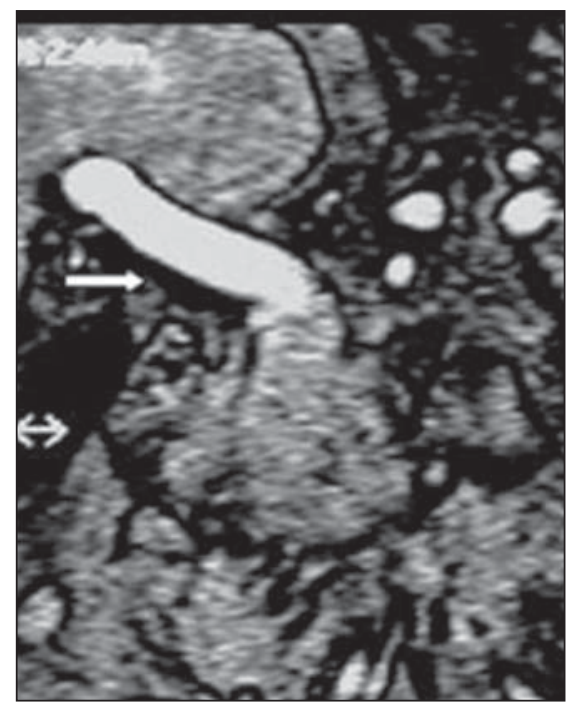

Figura 12. Visualização da veia porta (seta).

Figura 14. Imagem axial ponderada em T1 demonstrando varicosidades na porta hepatis (seta).

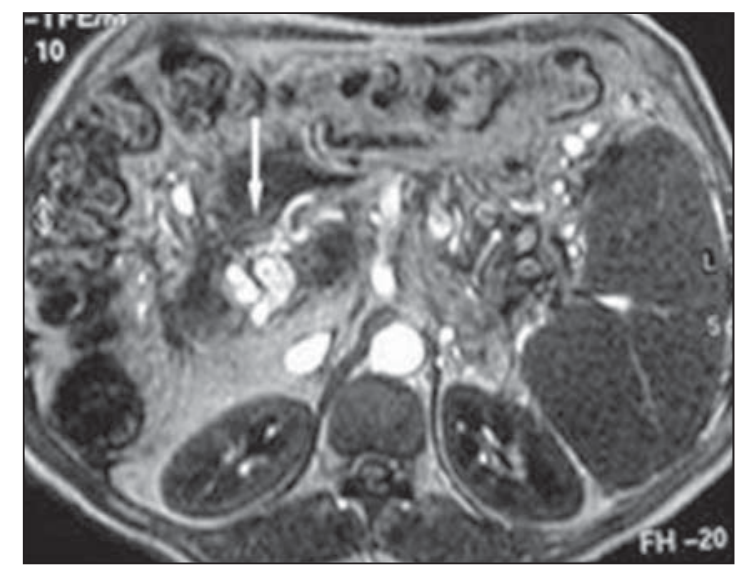

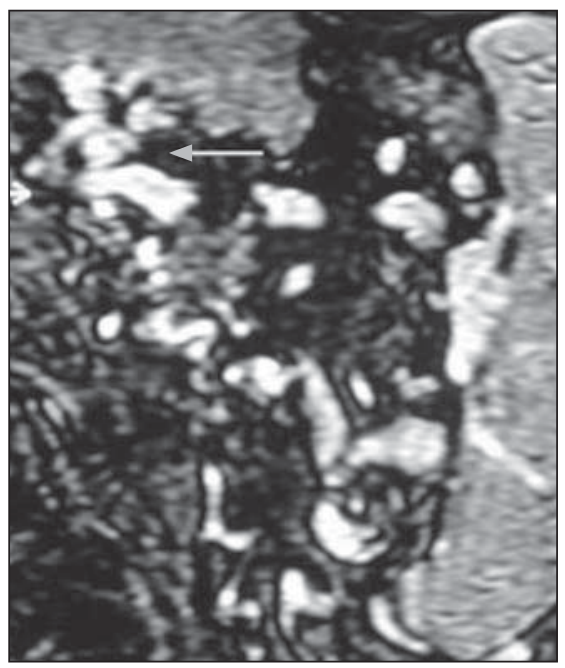

A

Figura 15. Imagens coronais da seqüência dinâmica tridimensional pós-contraste, com a demonstração de imagens vasculares serpiginosas no leito portal características da transformação cavernosa (seta). Notar a volumosa esplenomegalia decorrente da hipertensão portal. 
Tabela 1 Distribuição percentual dos graus de visualização dos segmentos vasculares para o grupo dose simples de contraste $(n=15)$.

\begin{tabular}{|l|c|c|c|}
\hline Segmento vascular & Grau 0 & Grau 1 & Grau 2 \\
\hline Tronco celíaco & 0 & 0 & 100 \\
Artéria hepática comum & 0 & 6,6 & 93,4 \\
Artéria hepática própria & 0 & 40 & 60 \\
Artéria gástrica esquerda & 0 & 73,3 & 26,7 \\
Artéria esplênica & 13,3 & 0 & 86,7 \\
Artéria gastroduodenal & 33,3 & 53,3 & 13,4 \\
Artéria gástrica direita & 93,3 & 6,7 & 0 \\
Artéria hepática direita & 33,3 & 33,3 & 33,4 \\
Artéria hepática esquerda & 33,3 & 40 & 26,7 \\
Artéria hepática média & 100 & 0 & 0 \\
Croça da artéria mesentérica superior & 0 & 0 & 100 \\
Artéria mesentérica superior, terço médio & 0 & 6,6 & 93,4 \\
Artéria mesentérica superior, terço distal & 6,6 & 46,7 & 46,7 \\
Artérias jejunais e ileais & 6,6 & 60 & 33,4 \\
Artéria cólica média & 80 & 20 & 0 \\
Artéria cólica direita & 33,3 & 60 & 6,7 \\
Artéria ileocólica & 13,3 & 60 & 26,7 \\
Artéria pancreaticoduodenal & 100 & 0 & 0 \\
Artéria mesentérica inferior & 20 & 60 & 20 \\
Veia porta & 0 & 26,7 & 73,3 \\
Ramo portal direito & 13,3 & 60 & 13,4 \\
Ramo portal esquerdo & 0,6 & 53,4 & 40 \\
Veia esplênica & 13,3 & 6,7 & 80 \\
Veia mesentérica superior & 0 & 13,3 & 86,7 \\
Veia mesentérica inferior & 0 & 86,7 \\
\hline
\end{tabular}

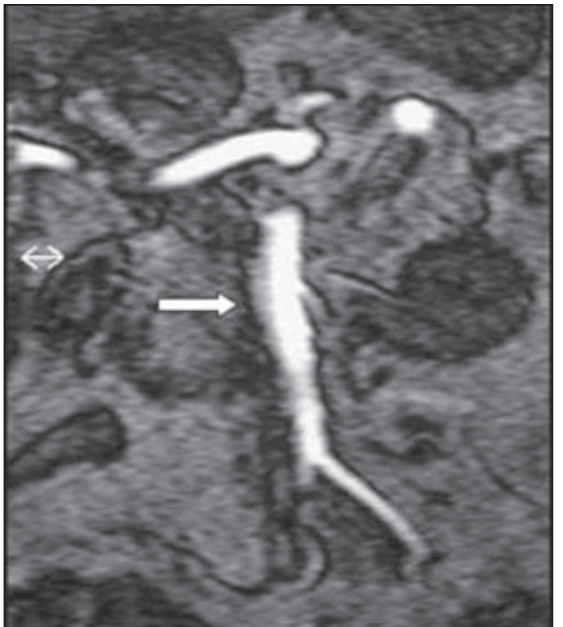

A

Figura 16. Comparação da visualização da artéria mesentérica superior (setas) pela angio-RM com contraste em pacientes diferentes com o uso de dose simples (A) e dose dupla (B) de gadolínio.

hepática direita teve maior frequiência do grau de visualização 2 com a dose simples. No entanto, foram poucos os casos responsáveis por essa diferença (cinco casos discordantes).

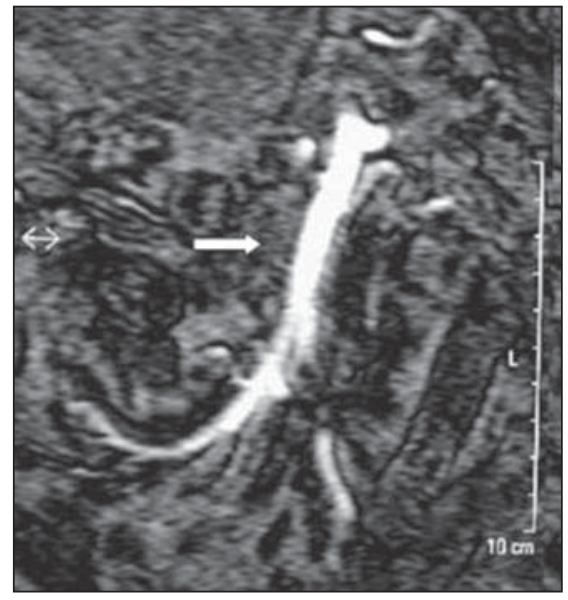

B

\section{DISCUSSÃO}

Nosso intuito, com este trabalho, não foi a correlação de métodos diagnósticos, uma vez que já foi feita a comparação entre a
angio-RM mesentérica e a arteriografia digital para o território em estudo ${ }^{(\mathbf{1 0})}$. Porém, os achados aqui descritos complementam as conclusões do trabalho desenvolvido por Ernst et al. ${ }^{(10)}$, em que houve boa correlação entre esses dois métodos na avaliação das porções proximais das artérias mesentéricas, com adequada visualização das ramificações distais obtida apenas na $\operatorname{arteriografia}^{(\mathbf{1 0})}$.

Um dos principais fatores que merecem consideração é a amostra em estudo. $O$ fato de avaliar um grupo de pacientes esquistossomóticos traz os benefícios de já estabelecer parâmetros para doentes que poderão se beneficiar da metodologia diagnóstica estudada. Além das informações diagnósticas obtidas em cada caso, os exames realizados neste estudo responderam à demanda já existente pelo método na avaliação de pacientes esquistossomóticos.

A randomização da amostra foi feita para eliminar qualquer influência não controlada, capaz de inadvertidamente viciar a distribuição dos $\operatorname{casos}^{(22)}$. A assimetria nas dimensões dos grupos de diferentes dosagens foi gerada pelo processo de randomização; porém, isto não representou problema para o método estatístico empregado.

Pode-se aumentar a sensibilidade da angio-RM e conseguir resultados ainda melhores de detalhamento vascular através da vasodilatação esplâncnica. Neste sentido, em 1998, Hany et al. comprovaram que o estímulo calórico obtido por refeição de $475 \mathrm{kcal}$ precedendo o exame em $15 \mathrm{mi}$ nutos é capaz de melhorar a detecção das ramificações arteriais mesentéricas ${ }^{\mathbf{2 3 3}}$. Isto não foi possível no nosso estudo, devido à realização de colangiopancreatografia por RM no mesmo período de exame em parte dos pacientes. Portanto, optamos em não incorporar este aprimoramento para fins de homogeneização da amostra.

Apesar de publicações recentes defenderem o uso de bobinas de sinergia ${ }^{(24-26)} \mathrm{em}$ vez de bobinas de corpo, em estudo comparativo desenvolvido no mesmo equipamento por nós utilizado, as sequiências com bobina de sinergia apresentaram qualidade de imagem semelhante e razão sinal/ruído inferior àquelas realizadas com bobina de corpo $^{(27)}$

A qualidade de um estudo por angioRM com contraste depende em muito do 
Tabela 2 Distribuição percentual dos graus de visualização dos segmentos vasculares para o grupo dose dupla de contraste $(n=21)$.

\begin{tabular}{|l|c|c|c|}
\hline Segmento vascular & Grau O & Grau 1 & Grau 2 \\
\hline Tronco celíaco & 0 & 0 & 100 \\
Artéria hepática comum & 0 & 14,2 & 85,8 \\
Artéria hepática própria & 0 & 38,1 & 6,9 \\
Artéria gástrica esquerda & 9,6 & 57,1 & 33,3 \\
Artéria esplênica & 23,8 & 0 & 76,2 \\
Artéria gastroduodenal & 38,1 & 57,1 & 4,8 \\
Artéria gástrica direita & 100 & 0 & 0 \\
Artéria hepática direita & 38,1 & 61,9 & 0 \\
Artéria hepática esquerda & 61,9 & 28,5 & 9,6 \\
Artéria hepática média & 100 & 0 & 0 \\
Croça da artéria mesentérica superior & 0 & 0 & 100 \\
Artéria mesentérica superior, terço médio & 0 & 0 & 100 \\
Artéria mesentérica superior, terço distal & 4,7 & 38,1 & 57,2 \\
Artérias jejunais e ileais & 0 & 66,6 & 33,4 \\
Artéria cólica média & 57,1 & 33,3 & 9,6 \\
Artéria cólica direita & 38,1 & 42,9 & 19 \\
Artéria ileocólica & 28,6 & 19,1 & 52,3 \\
Artéria pancreaticoduodenal & 100 & 0 & 0 \\
Artéria mesentérica inferior & 9,5 & 76,2 & 14,3 \\
Veia porta & 0 & 9,5 & 90,5 \\
Ramo portal direito & 14,2 & 52,4 & 33,4 \\
Ramo portal esquerdo & 9,5 & 47,6 & 42,9 \\
Veia esplênica & 19 & 19 & 62 \\
Veia mesentérica superior & 0 & 4,8 & 95,2 \\
Veia mesentérica inferior & 0 & 14,2 & 85,8 \\
\hline
\end{tabular}

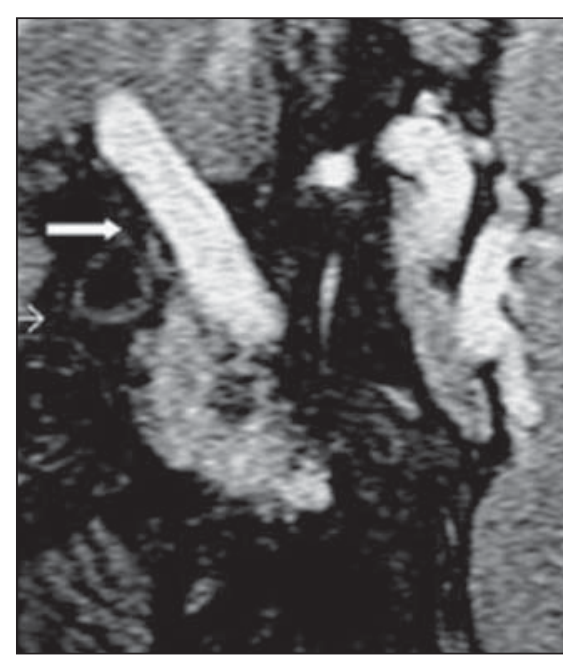

A

Figura 17. Comparação da visualização da veia porta (setas) pela angio-RM com contraste em pacientes diferentes com o uso de dose simples (A) e dose dupla (B) de gadolínio.

adequado planejamento temporal da fase dinâmica, assim como da técnica de injeção. A aquisição precoce dos dados resulta em insuficiente contrastação arterial, e a aquisição tardia causa a indesejável con-

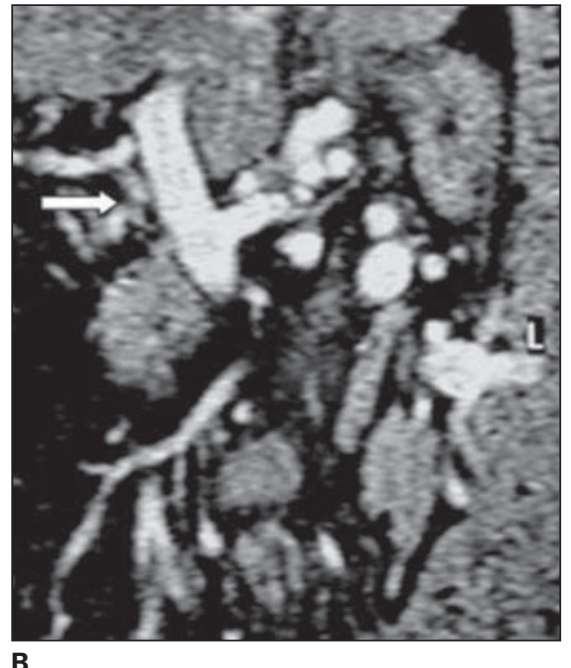

trastação venosa e realce de estruturas parenquimatosas. No presete estudo, a utilização da técnica da dose teste garantiu o adequado cálculo do intervalo de tempo para o início da sequiência dinâmica, mi- nimizando erros por falhas técnicas de planejamento temporal. Utilizamos a bomba injetora pelas vantagens de fornecer uma velocidade de injeção constante e padronizada, capaz de fornecer intensidade de sinal arterial superior àquela obtida com a injeção manual ${ }^{(28)}$.

\section{Freqüência de visualização dos segmentos vasculares}

De acordo com a análise dos resultados do grau de visualização dos ramos vasculares, é possível identificar três diferentes grupos vasculares: um grupo de segmentos muito bem visualizados, caracterizado pela alta freqüência de visualização grau 2; um grupo de segmentos de visualização intermediária, com distribuição dos resultados pelos três graus estabelecidos; e um terceiro grupo, constituído por segmentos de visualização insatisfatória, caracterizado pela alta frequiência da visualização grau 0 .

O primeiro grupo de segmentos vasculares (bem visualizados) é constituído pelo tronco celíaco, artéria hepática comum, artéria esplênica, croça e terço médio da artéria mesentérica superior, veia porta, veia esplênica e veia mesentérica superior. O segundo grupo (visualização intermediária) é constituído pelos seguintes segmentos vasculares: artérias hepáticas própria, direita e esquerda, artéria gástrica esquerda, artéria gastroduodenal, terço distal da artéria mesentérica superior, artérias jejunais e ileais, artérias cólicas média e direita, artéria ileocólica, artéria mesentérica inferior, ramos portais direito e esquerdo, e veia mesentérica inferior. E o terceiro grupo (visualização insatisfatória) é formado pela artéria gástrica direita, artéria hepática média e artéria pancreaticoduodenal inferior.

Observamos, portanto, que a adequada visualização dos segmentos vasculares apresentou correlação com o calibre e proximidade do trecho vascular em questão. De maneira geral e conforme o esperado, os segmentos proximais e de maior calibre foram identificados com maior nitidez que as ramificações distais. Estes achados são concordantes com as referências publicadas até o momento, com trabalhos demonstrando boa qualidade de imagem pela angio-RM para a avaliação do tronco celíaco e tronco arterial mesentérico, porém ruim 
para as artérias intra-hepáticas e ramificações mesentéricas ${ }^{(\mathbf{1 0})}$. Acreditamos que o pequeno calibre, a variabilidade anatômica e o grau de resolução espacial da angio-RM são os determinantes da insatisfatória visualização dos trechos vasculares distais por este método.

Se, por um lado, a avaliação do território arterial de pequeno calibre se mostrou insatisfatória, por outro, a adequada visualização dos dois terços proximais da artéria mesentérica superior é um excelente indicativo da potencialidade diagnóstica do método, pois a maioria das lesões ateroscleróticas, principal doença do leito arterial mesentérico, ocorre justamente nas porções proximais dessa artéria ${ }^{(29)}$.

De modo análogo, a boa visualização da maior extensão do leito venoso portomesentérico (veias porta, esplênica e mesentérica superior), trecho geralmente envolvido pelas doenças venosas da região, é um bom indicativo da potencial eficácia do método também para o território venoso.

Especificamente em relação à veia porta, é válido ressaltar que a presença de transformação cavernosa em alguns casos da amostra pode justificar sua parcial ou não visualização. Nestes casos, a menor visibilidade deste segmento vascular não ocorreu por limitação técnica e sim por alteração anatômica.

Os resultados obtidos também ilustram uma particularidade deste grupo de pacientes: a alta frequiência de trombose portal com transformação cavernosa, identificada em sete casos do total de 36 pacientes $(19,4 \%$ da amostra). O aspecto morfológico da transformação cavernosa da veia porta já foi bem estabelecido tanto pela $\mathrm{RM}^{(30)}$ como pela angio- $\mathrm{RM}^{(\mathbf{3 1})}$, e a sua identificação em nossa amostra é mais um indicativo da utilidade do método para esta finalidade.

\section{Comparação entre as doses simples e dupla de gadolínio na angio-RM}

Os resultados obtidos mostraram excelente equivalência na utilização das duas diferentes dosagens de gadolínio (simples e dupla). Não houve diferença significante no grau de visualização das diversas estruturas analisadas entre os grupos dose simples e dose dupla, exceto na avaliação da artéria hepática direita, em que o grupo dose simples apresentou maior frequiência de visualização grau 2, em valor significante.

De acordo com o esperado e a exemplo do observado em outros territórios vasculares, o uso da dose simples ou dupla de gadolínio forneceu resultados semelhantes no estudo do território vascular mesentérico. Portanto, a utilização da dose dupla de gadolínio não parece ser fator determinante de melhor visibilidade vascular, indicando que os resultados apresentados por Ernst et al. ${ }^{(\mathbf{1 0})}$, com dose simples de contraste, são representativos de uma metodologia adequada para a angio-RM mesentérica. Em nosso trabalho, apesar da utilização da técnica da dose teste, não conseguimos melhores índices de visualização que Ernst et al., que utilizaram múltiplas seqüências em intervalos de tempo pré-estabelecidos, o que sugere que ambos os métodos são úteis e adequados para o planejamento temporal.

Nossos resultados reforçam também os trabalhos publicados sobre estudos pela angio-RM com dose simples de contraste em outros territórios vasculares ${ }^{(14,15,17)}$.

Pode-se, portanto, admitir que é possível utilizar a dose simples de gadolínio no estudo da circulação hepatomesentérica pela angio-RM, com resultados comparáveis àqueles obtidos com a habitual dose dupla de contraste.

Os resultados do atual estudo reforçam o valor da angio-RM na avaliação da circulação hepatomesentérica, como uma alternativa segura, de fácil execução, disponibilidade crescente, e com elevada freqüência de visualização vascular, principalmente útil na investigação do leito proximal, maior sítio das alterações patológicas destes vasos.

Especificamente em relação ao paciente esquistossomótico, a angio-RM pode desempenhar papel fundamental nas reavaliações da circulação hepatomesentérica, principalmente em controles pós-operatórios. Em publicação recente, Lopes Filho e Haddad ressaltaram a importância de estudos evolutivos para avaliação da patência da anastomose cirúrgica em pacientes esquistossomóticos ${ }^{(32)}$. A utilidade da angio-RM, nesse sentido, é um dos diversos meios de se ampliar os benefícios de um método tão promissor.

\section{CONCLUSÕES}

A análise dos resultados obtidos a partir da avaliação da circulação hepatomesentérica através do exame de angio-RM com contraste em 36 pacientes esquistossomóticos permitiu concluir que:

1. A frequiência de visualização dos segmentos vasculares do leito hepatomesentérico é elevada, principalmente nos vasos proximais e de maior calibre.

2. A freqüência de visualização dos segmentos da circulação hepatomesentérica é semelhante ao compararmos o uso de dose simples e dose dupla de contraste paramagnético.

\section{REFERÊNCIAS}

1. Prince MR. Gadolinium-enhanced MR aortography. Radiology 1994;191:155-164.

2. Prince MR, Narasimham DL, Stanley JC, et al. Breath-hold gadolinium-enhanced MR angiography of the abdominal aorta and its major branches. Radiology 1995;197:785-792.

3. Hany TF, Debatin JF, Leung DA, Pfammatter T Evaluation of the aortoiliac and renal arteries: comparison of breath-hold, contrast-enhanced, three-dimensional MR angiography with conventional catheter angiography. Radiology 1997;204: 357-362.

4. D'Ippolito G, Wolosker N, Galvão Filho M, Kalil JA, Wolosker A, Borri ML. Angio-RM com contraste no estudo das doenças da aorta tóracoabdominal. Rev Imagem 1998;20:85-93.

5. D'Ippolito G, Wolosker N, Galvão MM, Wolosker AMB, Borri ML. Angio-RM com contraste e angiografia convencional na avaliação do paciente com claudicação: estudo comparativo. Rev Angiol 1999;15:78-85.

6. Wolosker N, Nakano L, D'Ippolito G, Rosoky RMA, Borri ML, Wolosker AMB. Gadolinium magnetic angioresonance in the study of aortoiliac disease. Angiology 2003;54:163-168.

7. Thornton MJ, Thornton F, O'Callaghan J, et al. Evaluation of dynamic gadolinium-enhanced breath-hold MR angiography in the diagnosis of renal artery stenosis. AJR Am J Roentgenol 1999; 173:1279-1283.

8. Huston J 3rd, Fain SB, Wald JT, et al. Carotid artery: elliptic centric contrast-enhanced MR angiography compared with conventional angiography. Radiology 2001;218:138-143.

9. Wutke R, Lang W, Fellner C, et al. High-resolution, contrast-enhanced magnetic resonance angiography with elliptical centric k-space ordering of supra-aortic arteries compared with selective X-ray angiography. Stroke 2002;33:1522-1529.

10. Ernst O, Asnar V, Sergent G, et al. Comparing contrast-enhanced breath-hold MR angiography and conventional angiography in the evaluation of mesenteric circulation. AJR Am J Roentgenol 2000;174:433-439.

11. Davis CP, Hany TF, Wildermuth S, Schmidt M, Debatin JF. Postprocessing techniques for gadolinium-enhanced three-dimensional MR angiography. RadioGraphics 1997;17:1061-1077. 
12. Lentschig MG, Reimer P, Rausch-Lentschig UL, Allkemper T, Oelerich M, Laub G. Breath-hold gadolinium-enhanced MR angiography of the major vessels at $1.0 \mathrm{~T}$ : dose-response findings and angiographic correlation. Radiology 1998;208: 353-357.

13. Thurnher SA, Capelastegui A, Del Olmo FH, $e$ al. Safety and effectiveness of single- versus triple-dose gadodiamide injection- enhanced MR angiography of the abdomen: a phase III doubleblind multicenter study. Radiology 2001;219: 137-146.

14. Lee VS, Rofsky NM, Krinsky GA, Stemerman DH, Weinreb JC. Single-dose breath-hold gadolinium-enhanced three-dimensional MR angiography of the renal arteries. Radiology 1999;211: 69-78.

15. Korst MB, Joosten FB, Postma CT, Jager GJ, Krabbe JK, Barentsz JO. Accuracy of normaldose contrast-enhanced MR angiography in assessing renal artery stenosis and accessory renal arteries. AJR Am J Roentgenol 2000;174:629_ 634.

16. Volk M, Strotzer M, Lenhart M, et al. Renal timeresolved MR angiography: quantitative comparison of gadobenate dimeglumine and gadopentetate dimeglumine with different doses. Radiology 2001;220:484-488.

17. Hartmann LG, Wolosker AMB, D'Ippolito G, Borri ML, Kalil J. Angio-RM das artérias carótidas e vertebrais: análise de diferentes técnicas de volume e diluição de contraste em aparelho de 1,0 $\mathrm{T}$ e gradiente de15 mT/m. Radiol Bras 2001;34 201-205.
18. Maki JH, Wilson GJ, Eubank WB, Hoogeveen RM. Utilizing SENSE to achieve lower station sub-millimeter isotropic resolution and minimal venous enhancement in peripheral MR angiography. J Magn Reson Imaging 2002;15:484-491.

19. Willinek WA, Gieseke J, Conrad R, et al. Randomly segmented central k-space ordering in high-spatial-resolution contrast-enhanced MR angiography of the supraaortic arteries: initial experience. Radiology 2002;225:583-588.

20. Altman DG, Bland JM. How to randomise. BMJ 1999;319:703-704.

21. Altman DG, Bland JM. Statistics notes. Treatment allocation in controlled trials: why randomise? BMJ 1999;318:1209.

22. Hall JC, Hall JL. Randomization in surgical trials. Surgery 2002;132:513-518.

23. Hany TF, Schmidt M, Schoenenberger AW, Debatin JF. Contrast-enhanced three-dimensional magnetic resonance angiography of the splanchnic vasculature before and after caloric stimulation. Original investigation. Invest Radiol 1998; 33:682-686.

24. Campeau NG, Johnson CD, Felmlee JP, et al. MR imaging of the abdomen with a phased-array multicoil: prospective clinical evaluation. Radiology 1995;195:769-776.

25. Schwartz LH, Panicek DM, Thomson E, et al. Comparison of phased-array and body coils for MR imaging of liver. Clin Radiol 1997;52:745749

26. Huber A, Scheidler J, Wintersperger B, et al. Moving-table MR angiography of the peripheral runoff vessels: comparison of body coil and dedicated phased array coil systems. AJR Am J Roentgenol 2003;180:1365-1373.

27. Lima CA. Estudo comparativo das sequiências rápidas ponderadas em $\mathrm{T} 2$ utilizando sincronização respiratória, apnéia, supressão de gordura e bobina de sinergia para a avaliação do fígado pela ressonância magnética. (Tese de Doutorado). São Paulo: Unifesp-EPM, 2002.

28. Kopka L, Vosshenrich R, Rodenwaldt J, Grabbe E. Differences in injection rates on contrast-enhanced breath-hold three-dimensional MR angiography. AJR Am J Roentgenol 1998; 170:345348.

29. Gaa J, Georgi M. Non-invasive imaging of abdominal vascular pathologies. Eur Radiol 1998;8: 507-516.

30. Ros PR, Viamonte M Jr, Soila K, Sheldon JJ, Tobias J, Cohen B. Demonstration of cavernomatous transformation of the portal vein by magnetic resonance imaging. Gastrointest Radiol 1986;11: 90-92.

31. Caldana RP, Bezerra ASA, Palácio GAS, D'Ippolito G, Szejnfeld J. Transformação cavernomatosa da veia porta: caracterização pela angiografia por ressonância magnética com gadolínio. Radiol Bras 2002;35(Supl 1):9.

32. Lopes Filho GJ, Haddad CM. Late clinical, biochemical, endoscopic and electroencephalographic evaluation of patients with schistosomal portal hypertension treated with distal splenorenal shunt. Int Surg 1998;83:42-47. 